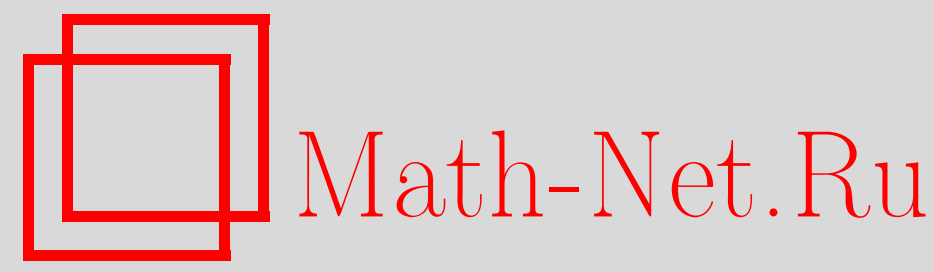

В. М. Бухштабер, К. Э. Фельдман, Индекс эквивариантного векторного поля и теоремы сложения для характеристических классов Понтрягина, Изв. РАН. Сер. матем., 2000, том 64, выпуск 2, 3-28

DOI: https://doi.org/10.4213/im282

Использование Общероссийского математического портала Math-Net.Ru подразумевает, что вы прочитали и согласны с пользовательским соглашением http: //www. mathnet.ru/rus/agreement

Параметры загрузки:

IP : 54.157 .27 .8

26 апреля 2023 г., 12:56:32 
УДК 515.145

\author{
В. М. Бухштабер, К. Э. Фельдман
}

\title{
Индекс эквивариантного векторного поля и теоремы сложения для характеристических классов Понтрягина
}

\begin{abstract}
В работе построена теория индексов векторных полей Морса-Ботта на многообразии и на ее основе решена известная задача о локализации трансфера гладких расслоений. В качестве следствия получены теоремы сложения для классов Понтрягина вешественных векторных расслоений в кобордизмах. Это позволило завершить построение теории характеристических классов, начатое более двадцати лет назад.
\end{abstract}

Библиографияя: 20 наименований.

\section{§1. Введение}

Конструкция трансфера расслоений [2], [3] является одним из наиболее фундаментальных понятий в современной алгебраической топологии. Она позволила существенно развить и расширить области приложений метода прямого образа, связанного с такими понятиями, как прямой образ представлений, след алгебраического расширения, трансфер векторных расслоений для накрытий, гомоморфизм Гизина и т.д. (см. [1]-[6], [10]-[12], [17]).

В настоящей работе построена теория индексов векторных полей Морса-Ботта на многообразии и на ее основе решена известная задача о локализации трансфера гладких расслоений. Этот результат является далеким обобшением классической теоремы Пуанкаре-Хопфа, выражающей эйлерову характеристику многообразия в виде суммы индексов нулей касательного векторного поля общего положения.

В качестве следствия для универсальных (т.е. со значениями в теориях кобордизмов) классов Понтрягина вещественных векторных расслоений получены формулы, выражающие характеристические классы Понтрягина суммы расслоений через характеристические классы слагаемых (теоремы сложения). Это позволило завершить построение теории универсальных характеристических классов, начатое более двадцати лет назад [6].

Изложим кратко содержание статьи. В $\S 2$ мы напоминаем конструкцию трансфера для гладких расслоений и соответствующее определение индекса расслоения. В $\S 3$ вводится индекс $\operatorname{Ind}_{s}\left(F_{l}\right)$ векторного поля Морса-Ботта $s$ на гладком многообразии $F$ в окрестности подмногообразия нулей $F_{l}$. Этот индекс принимает значение в когомотопической группе $\pi_{S}^{0}\left(F_{l}^{+}\right)$. Приводится пример векторного поля Морса-Ботта на проективной плоскости, у которого индекс в окрестности проективной прямой равен $-1+u$, где $u$ - образуюшая группы $\pi_{1}^{S}=Z_{2}$. Доказывается важная теорема 3.1 локализации трансфера многообразия (как расслоения

Работа выполнена при финансовой поддержке РФФИ (грант № 99-01-00090).

(C) В. М. БУХШТАБЕР, К.Э. ФЕЛЬДМАн, 2000 
над точкой) при помощи векторного поля Морса-Ботта на нем. В качестве следствия для векторного поля Морса-Ботта $s$ с набором связных подмногообразий нулей $\left\{F_{l}\right\}$ получено выражение эйлеровой характеристики $\chi(F)$ через эйлеровы характеристики $\chi\left(F_{l}\right)$ и индексы $\operatorname{Ind}_{s}\left(F_{l}\right)$ (теорема 3.2 ). В $\S 4$ доказывается теорема о локализации трансфера в обшем случае для произвольного расслоения с гладким слоем. Показано, что известные результаты [5], [11] в этом направлении сводятся к ее частным случаям.

Оставшаяся часть работы посвяшена приложениям полученных результатов к теории характеристических классов вешественных векторных расслоений. В $\S 5$ построено важное поле Морса-Ботта на грассманизации суммы Уитни двух вещественных векторных расслоений. Описаны связные компоненты подмногообразий нулей и вычислены индексы этого поля в их окрестностях.

В $\S 6$ получен один из центральных результатов работы - теорема сложения универсальных классов Понтрягина вещественных векторных расслоений. Детально рассмотрен случай первого полуцелого класса $p_{1 / 2}$. Показано, что на образе отображения

$$
p_{1 / 2}: K O^{*}(X) \rightarrow U^{2}(X)
$$

возникает новая алгебраическая операция, не сводимая ни к какой формальной группе. Обратим внимание, что в нашей краткой заметке [9] в формуле для $p_{1 / 2}(\xi \oplus \zeta)$ в комплексных кобордизмах была допушена неточность, которую мы исправляем в настоящей работе.

В последнем параграфе исследуются классы Понтрягина стабильно комплексных векторных расслоений. Показано, что если вещественное векторное расслоение допускает стабильную комплексную структуру, то его классы Понтрягина с полуцелыми номерами равны нулю, а классы Понтрягина с целыми номерами обладают реализацией в симплектических кобордизмах.

Авторы выражают глубокую признательность И. А. Дьнникову за полезные обсуждения и ценные замечания в ходе выполнения этой работы.

\section{§2. Индекс гладкого расслоения}

В этом параграфе мы дадим определения основных понятий, связанных с трансфером Беккера-Готтлиба [3].

Пусть $X$ и $Y$ - топологические пространства с отмеченными точками и $[X, Y]-$ множество гомотопических классов отображений $X$ в $Y$, переводящих отмеченную точку в отмеченную. Обозначим через $\{X, Y\}$ группу стабильных гомотопических классов отображений

$$
\{X, Y\}=\lim _{n \rightarrow \infty}\left[S^{n} \wedge X, S^{n} \wedge Y\right] .
$$

ОПРЕДЕЛЕНИЕ 2.1. Нульмерной когомотопической группой пространства $X$ с отмеченной точкой называется группа

$$
\pi_{S}^{0}(X)=\left\{X, S^{0}\right\}=\lim _{n \rightarrow \infty}\left[S^{n} \wedge X, S^{n}\right]
$$


Будем обозначать через $X^{+}$несвязное объединение пространства $X$ и точки, которую будем полагать отмеченной. Если в пространстве $X$ задана отмеченная точка, то $S^{n} \wedge X^{+}$гомотопически эквивалентно $S^{n} X \vee S^{n}$, и поэтому

$$
\pi_{S}^{0}\left(X^{+}\right)=\pi_{S}^{0}(X) \oplus Z
$$

Каноническую проекцию $\pi_{S}^{0}\left(X^{+}\right) \rightarrow Z$ мы будем обозначать через $\epsilon$. Если в качестве $X$ взять сферу $S^{k}$, то

$$
\operatorname{Ker}(\epsilon) \cong \pi_{k}^{S}
$$

где $\pi_{k}^{S}-k$-я стабильная гомотопическая группа сфер.

Каждое расслоение $(E, F, B, p)$ с гладким компактным замкнутым слоем $F$ задает канонический элемент в групе $\left\{B^{+}, E^{+}\right\}$, существование которого обеспечивается трансфером Беккера-Готтлиба [3]. Приведем конструкцию этого элемента в случае, когда $E$ есть гладкое расслоение с компактной базой (для произвольных клеточных баз см. определение в [8]).

Пространство расслоения $E$ может быть вложено в $\mathbb{R}^{n}$ для подходящего $n$. Обозначим это вложение как $i$. Пусть $\tau_{F}(E)$ - касательное расслоение вдоль слоев расслоения $E$ и $\nu(E)$ - нормальное расслоение вложения $p \times i: E \subset B \times \mathbb{R}^{n}$. Нетрудно проверить, что $\tau_{F}(E) \oplus \nu(E)$ есть тривиальное $n$-мерное векторное расслоение над $E$ [3]. Будем обозначать пространство Тома произвольного векторного расслоения $\xi$ как $T \xi$.

ОПРЕДЕЛЕНИЕ 2.2. Трансфером гладжого расслоения $(E, F, B, p)$ называется композиция отображений

$$
\tau(p): S^{n} \wedge B^{+} \rightarrow T \nu(E) \rightarrow T\left(\nu(E) \oplus \tau_{F}(E)\right)=T[\bar{n}],
$$

где первое отображение - это отображение Понтрягина-Тома, представляющее собой склейку внешности трубчатой окрестности подмногообразия $E$ в многообразии $B \times \mathbb{R}^{n}$, а второе отображение есть вложение расслоения $\nu(E)$ на прямое слагаемое.

Заметим, что пространство Тома тривиального $n$-мерного векторного расслоения над $B$ есть $n$-кратная надстройка над $B^{+}$. Поэтому трансфер определяет некоторый элемент группы $\left[S^{n} \wedge B^{+}, S^{n} \wedge E^{+}\right]$. Образ этого элемента в группе $\left\{B^{+}, E^{+}\right\}$уже не зависит от выбора вложения [3]. Таким образом, трансфер корректно определяет элемент группы $\left\{B^{+}, E^{+}\right\}$, который мы будем обозначать через $\{\tau(p)\}$.

Стабильное отображение из $X$ в $Y$ для любой теории когомологий $h^{*}(\cdot)$ однозначно определяет гомоморфизм групा

$$
h^{*}(Y) \rightarrow h^{*}(X)
$$

Соответствуюший гомоморфизм для трансфера расслоения $(E, F, B, p)$ мы будем обозначать через $\tau(p)^{*}$. Пусть $\pi$-проекция на первое слагаемое $\pi: S^{n} \wedge E^{+} \rightarrow S^{n}$. 
ОПРЕДЕЛЕНИЕ 2.3. Индексом $I(E)$ расслоения $p: E \rightarrow B$ называется элемент $\{\pi \circ \tau(p)\} \in \pi_{S}^{0}\left(B^{+}\right)$. Индексом этого расслоения в теории когомологий $h^{*}(\cdot)$ называется элемент $I_{h}(E)=\tau(p)^{*}(1) \in h^{0}\left(B^{+}\right)$(мы считаем, что в $h^{*}$ группа $h^{0}$ является кольцом с единицей). Таким образом, $I(E)$ - индекс расслоения в стабильной теории когомотопий $\pi_{S}^{*}(\cdot)$.

Заметим, что для любой теории когомологий $h^{*}(\cdot)$ с единицей существует каноническое преобразование теорий (см. [19])

$$
\mu_{h}: \pi_{S}^{*}(\cdot) \rightarrow h^{*}(\cdot)
$$

которое переводит единицу теории $\pi_{S}^{*}(\cdot)$ в единицу теории $h^{*}(\cdot)$. Индекс расслоения $p: E \rightarrow B$ в теории $h^{*}(\cdot)$ равен $\mu_{h}(I(E))$.

Индексы расслоений нетривиальны уже в простейшем случае для расслоений, база которых есть точка. В этом случае $\pi_{S}^{0}\left(B^{+}\right) \cong Z$, и индекс расслоения равен эйлеровой характеристике слоя [3].

\section{§3. Локализация}

Фундаментальной характеристикой невырожденного нуля касательного векторного поля на гладком многообразии является индекс этого векторного поля в окрестности рассматриваемого нуля [14]. Индекс векторного поля определяется как знак детерминанта линейной части векторного поля в малой окрестности нуля [14]. В этом параграфе мы покажем, что в окрестности вырожденного нуля дифференциал поля дает важную характеристику этого нуля. В терминах вводимых характеристик выражаются коэффициенты, с которыми трансфер гладкого многообразия может быть представлен в виде линейной комбинации трансферов подмногообразий нулей векторного поля на исходном многообразии.

Прежде чем приступить к доказательству основной теоремы, мы приведем пример, который поможет понять формулировку свойства локализации трансфера. Начнем с необходимых понятий.

Каждый элемент группы $\pi_{S}^{0}\left(B^{+}\right)$задается непрерывным отображением вида $S^{n} \wedge B^{+} \rightarrow S^{n}$ для достаточно большого $n$. Обозначим через $\pi$ проекцию на первый множитель в пространстве $S^{n} \wedge B^{+}$. Представитель $\gamma$ элемента из $\pi_{S}^{0}\left(B^{+}\right)$ задает отображение

$$
f_{\gamma}: B \rightarrow \operatorname{Map}\left(S^{n}, S^{n}\right)
$$

по формуле $f_{\gamma}(b)(y)=\gamma(y, b)$. Верно и обратное. Любое непрерывное отображение $f: B \rightarrow \operatorname{Map}\left(S^{n}, S^{n}\right)$ задает некоторый элемент из $\pi_{S}^{0}\left(B^{+}\right)$по формуле $\gamma_{f}=\{\pi(f(b)(y), b)\}$. Отождествим $S^{n}$ c $\mathbb{R}^{n} \cup\{\infty\}$ и определим действие $\mathrm{GL}(n, \mathbb{R})$ на $S^{n}$ посредством этого отождествления. Уже в случае, когда $f(b) \in \mathrm{GL}(n, \mathbb{R})$ для любого $b \in B$, соответствуюший элемент когомотопической групшы может иметь ненулевую компоненту в $\operatorname{Ker}(\epsilon)$.

ПримеР 3.1. Пусть $B=S^{1}$ и $\xi_{1}$ - нетривиальное одномерное вешественное векторное расслоение над $S^{1}$. Заметим, что $\xi_{1} \oplus \xi_{1}$ - тривиальное двумерное вещественное векторное расслоение. Рассмотрим отображение

$$
S^{2} \wedge S^{1+}=T\left(\xi_{1} \oplus \xi_{1}\right) \stackrel{(1,-1)}{\longrightarrow} T\left(\xi_{1} \oplus \xi_{1}\right)=S^{2} \wedge S^{1+} \stackrel{\pi}{\longrightarrow} S^{2} .
$$


Так как $S^{2} \wedge S^{1+} \sim S^{3} \vee S^{2}$, то построенное вьше отображение определяет два отображения $\alpha_{1}: S^{3} \rightarrow S^{2}$ и $\alpha_{2}: S^{2} \rightarrow S^{2}$. Нетрудно проверить, что $\alpha_{2}$ есть симметрия относительно центральной плоскости. Покажем, что $\alpha_{1}$ задает образующую группы $\pi_{3}\left(S^{2}\right)$. Отождествим $S^{2}$ и $\mathbb{C}^{1} \cup\{\infty\}$, полагая бесконечно удаленную точку отмеченной. Рассмотрим прообраз нуля при отображении $\alpha_{1}$. Это будет нулевое сечение в расслоении $\xi_{1} \oplus \xi_{1}$, т.е. окружность. Полньй прообраз $\mathbb{C}^{1} \subset S^{2}$ при отображении $\alpha_{1}$ представляет собой пространство расслоения $\xi_{1} \oplus \xi_{1}$. Расщепление тривиального двумерного вешественного расслоения над $S^{1}$ в прямую сумму $\xi_{1} \oplus \xi_{1}$ задает выделенный базис в каждом слое расслоения

$$
\left\langle e_{1}(\phi), e_{2}(\phi)\right\rangle=\left\langle e^{i \phi / 2}, i e^{i \phi / 2}\right\rangle,
$$

где $\phi \in[0,2 \pi]$ - угловая координата на нулевом сечении (т.е. на $\left.S^{1}\right)$. Для каждой точки $\{\phi, x+i y\}$ из $S^{1} \times \mathbb{C}^{1} \subset S^{3}$ ее образ при отображении $\alpha_{1}$ есть

$$
\alpha_{1}(\{\phi, x+i y\})=\left\{(x-i y) e^{i \phi}\right\} \in \mathbb{C}^{1} .
$$

Отображение $\alpha_{1}$ в координатах $\left(z_{1}, z_{2}\right) \in \mathbb{C}^{1} \times \mathbb{C}^{1},\left|z_{1}\right|=1$, выражается по формуле

$$
\alpha_{1}\left(z_{1}, z_{2}\right)=\bar{z}_{2} z_{1},
$$

которая показывает, что отображение $\alpha_{1}$ гомотопно проекции в расслоении Хопфа.

Следовательно, соответствуюший отображению (3.1) элемент из $\pi_{S}^{0}\left(S^{1+}\right)$ есть $-1+u$, где $u$ - образуюшая группы $\pi_{1}^{S} \cong Z_{2}$.

Теперь мы перейдем к формулировке свойства локализации, которое сначала будет доказано для гладких многообразий, т.е. в случае расслоений, база которых есть точка.

Нам потребуется понятие касательного векторного поля Морса-Ботта (см. [16]), частным случаем которого являются морсовские векторные поля.

ОПРЕДЕЛЕНИЕ 3.1. Касательное векторное поле на гладком многообразии $F$ называется полем Морса-Ботта, если вьполняются следуюшие два условия:

1) нули векторного поля образуют конечньй набор связных компактных замкнутых подмногообразий в $F$;

2) в точках подмногообразия нулей ядро матрицы Якоби векторного поля совпадает с касательным пространством к подмногообразию нулей.

Рассмотрим компоненту связности $F_{l}$ нулей векторного поля Морса-Ботта. Обозначим через $\nu_{l}$ нормальное расслоение вложения $F_{l} \subset F$. Отметим, что пространство расслоения $\nu_{l}$ может быть отождествлено посредством экспоненциального отображения с трубчатой окрестностью подмногообразия $F_{l}$ в $F$ [14]. Ограничение векторного поля Морса-Ботта на нормальную трубку задает векторное поле на пространстве $\nu_{l}$. В силу условия 2) последнее векторное поле обязано иметь всюду ненулевую проекцию на касательное пространство вдоль слоев расслоения $\nu_{l}$ в достаточно малой окрестности нулевого сечения. Поэтому без ограничения обшности можно считать, что построенное на пространстве $\nu_{l}$ векторное поле всюду является касательным вдоль слоев расслоения $\nu_{l}$. Обозначим это векторное поле через $s_{l}$ :

$$
s_{l}: \nu_{l} \rightarrow \hat{\tau}\left(\nu_{l}\right)
$$


где $\hat{\tau}\left(\nu_{l}\right)$ - касательное расслоение вдоль слоев. Пусть $p$ - проекция в расслоении $\nu_{l}$; тогда $\hat{\tau}\left(\nu_{l}\right) \cong p^{*} \nu_{l}$ (см., например, [13]). Таким образом, векторное поле $s_{l}$ позволяет нам построить отображение $\psi_{l}^{s}: \nu_{l} \rightarrow \nu_{l}$ по правилу

$$
\psi_{l}^{s}(v)=\hat{p} s(v),
$$

где $\hat{p}$ - отображение расслоений $\hat{p}: p^{*} \nu_{l} \rightarrow \nu_{l}$.

Рассмотрим гладкое компактное замкнутое многообразие $F$ и некоторое векторное поле Морса-Ботта $s$ на нем. Пусть $F_{1}, \ldots, F_{m}$ - набор связных компактных замкнутых подмногообразий нулей поля $s$ на $F$. Фиксируем вложение многообразия $F$ в $n$-мерное евклидово пространство:

$$
F_{1}, \ldots, F_{m} \subset F \subset \mathbb{R}^{n} .
$$

Сопоставим при помощи векторного поля $s$ каждому подмногообразию $F_{l}$ отображение

$$
j_{l}: S^{n} \wedge F_{l}^{+} \rightarrow S^{n} \wedge F_{l}^{+}
$$

следуюшим образом. Вложение подмногообразий $F_{l} \subset F \subset \mathbb{R}^{n}$ индуцирует разложение тривиального $n$-мерного векторного расслоения над $F_{l}$ в прямую сумму касательного расслоения $\tau_{l}$ к $F_{l}$, нормального расслоения $\nu_{l}$ вложения $F_{l} \subset F$ и ограничения на $F_{l}$ нормального расслоения $\nu$ вложения $F \subset \mathbb{R}^{n}$. Поле $s$ задает отображение $\phi_{l}^{s}: \nu_{l} \rightarrow \nu_{l}$. Определим отображение $j_{l}$ на подрасслоениях $\nu$ и $\tau_{l}$ тождественным, а на $\nu_{l}$ равным $\phi_{l}^{s}$.

Как и выше, обозначим через $\pi$ проекцию $S^{n} \wedge F_{l}^{+} \rightarrow S^{n}$ на первый сомножитель.

ОПРЕДЕЛЕНИЕ 3.2. Элемент $\left\{\pi \circ j_{l}\right\} \in \pi_{S}^{0}\left(F_{l}^{+}\right)$будем назьвать индексом $\operatorname{Ind}_{s}\left(F_{l}\right)$ векторного поля $s$ в окрестности подмногообразия нулей $F_{l}$.

ЗАмЕЧАНИЕ 3.1. Индекс изолированного нуля векторного поля совпадает с $\epsilon\left(\operatorname{Ind}_{s}(p t)\right)$ и равен обычному индексу изолированного нуля [14].

ПРИмеР 3.2. Используя пример 3.1, покажем, что индекс $\operatorname{Ind}_{s}\left(F_{l}\right) \in \pi_{s}^{0}\left(F_{l}^{+}\right)$ может иметь ненулевую компоненту в $\operatorname{Ker}(\epsilon)$.

Определим действие группы положительных чисел $\mathbb{R}_{+}$на $R P^{2}$ по формуле

$$
t \circ\left(x_{1}: x_{2}: x_{3}\right)=\left(x_{1}: x_{2}: t^{-1} x_{3}\right) .
$$

Дифференциал этого действия при $t=1$ задает касательное векторное поле $s$ на $R P^{2}$. Нулями $s$ будут неподвижные точки действия, т.е.

$$
R P^{1}=\left\{\left(x_{1}: x_{2}: 0\right)\right\} \subset R P^{2}
$$

и точка $(0: 0: 1) \in R P^{2}$. Нормальное расслоение вложения $R P^{1} \subset R P^{2}$ можно отождествить с нетривиальным одномерным вещественным векторным расслоением $\xi_{1}$ над $S^{1}$. В окрестности $R P^{1}=S^{1} \subset R P^{2}$ векторное поле $s$ задается формулой $s(v)=-v, v \in \xi_{1} \approx N\left(S^{1}\right) \subset R P^{2}$. В силу примера 3.1 индекс $\operatorname{Ind}_{s}\left(R P^{1}\right)$ равен $-1+u$, где $u$ - образуюшая группы $\pi_{1}^{S} \cong Z_{2}$.

Пусть отображение $\tau_{l}$ есть трансфер многообразия $F_{l}$. Обозначим через $i$ вложение пространства Тома тривиального $n$-мерного векторного расслоения над $F_{1} \cup$ $\cdots \cup F_{m}$ в пространство Тома тривиального $n$-мерного векторного расслоения над $F$, соответствуюшее вложению

$$
F_{1} \cup \cdots \cup F_{m} \subset F .
$$


ТЕОРема 3.1 (свойство локализации). Трансфер $\tau$ многобразия $F$ гомотопен композичии

$$
i \circ\left(j_{1} \circ \tau_{1} \vee \cdots \vee j_{m} \circ \tau_{m}\right) \circ g,
$$

где $g$ - отображение, переводящее сферу $S^{n}$ в букет $m$ сфер.

ДоказАТЕЛьСтво. Вложение пространства Тома

$$
\alpha: T \nu(F) \rightarrow T(\nu(F) \oplus \tau(F))
$$

использованное нами при определении трансфера, можно заменить на гомотопное, воспользовавшись наличием касательного векторного поля. Именно, рассмотрим однопараметрическое семейство отображений

$$
\alpha_{t}(v)=\alpha(v) \oplus t s(\pi(v)), \quad v \in \nu(F),
$$

где $\pi$ - проекция в расслоении $\nu(F)$. Выбирая достаточно большой параметр $t$, мы получим гомотопное $\alpha$ отображение, которое переводит внешности малых окрестностей нулей поля $s$ в отмеченную точку пространства $T(\nu(F) \oplus \tau(F))$. Это означает, что отображение $\tau$ может быть пропущено через отображение $g$ и букет отображений, переводяших $S^{n}$ в $T \nu\left(F_{l}\right), l=1, \ldots, m$.

Для того чтобы описать устройство отображения $\tau$ в окрестности нулей поля $s$, нам будет удобно заменить отображение $\alpha$ на гомотопное, используя каноническое отождествление трубчатых окрестностей подмногообразий $F_{l}$ и нормальных расслоений вложений $F_{l} \subset F, l=1, \ldots, m$.

Обозначим через $\exp _{l}$ экспоненциальное отображение, которое задает диффеоморфизм расслоения на единичные диски, ассоциированного с нормальньм расслоением $\nu_{l}$ вложения $F_{l} \subset F$, и трубчатой окрестности $N\left(F_{l}\right) \subset F$ подмногообразия $F_{l}$ в $F, l=1, \ldots, m$. Для каждого $l=1, \ldots, m$ определим две функции

$$
\lambda_{l}, \mu_{l}: D^{k_{l}} \rightarrow \mathbb{R}^{k_{l}},
$$

где $D^{s}$ обозначает единичный диск размерности $s, k_{l}=\operatorname{codim} F_{l}$, полагая

$$
\lambda_{l}(v)= \begin{cases}|v|, & |v| \leqslant \frac{1}{2}, \\ 1-|v|, & |v|>\frac{1}{2},\end{cases}
$$

и

$$
\mu_{l}(v)= \begin{cases}0, & |v| \leqslant \frac{1}{2}, \\ 2|v|-1, & |v|>\frac{1}{2} .\end{cases}
$$

Рассмотрим отображение

$$
\tilde{\alpha}(x)= \begin{cases}\alpha(x), & x \notin N\left(F_{l}\right), \\ \left\{\exp \left(\mu_{l}\left(\exp _{l}^{-1}(x)\right) \exp _{l}^{-1}(x)\right), \lambda_{l}\left(\exp _{l}^{-1}(x)\right) \exp _{l}^{-1}(x)\right\}, & x \in N\left(F_{l}\right), \\ l=1, \ldots, m . & \end{cases}
$$


Поскольку $\lambda_{l}$ гомотопно нулевому отображению и $\mu_{l}\left(\exp _{l}^{-1}(x)\right) \exp _{l}^{-1}(x)$ гомотопно отображению $\exp ^{-1}(x)$, то отображение $\tilde{\alpha}$ гомотопно вложению на нулевое сечение $F \rightarrow \tau(F)$.

Заметим, что

$$
\left.\left.\nu(F)\right|_{N\left(F_{l}\right)} \cong \psi_{l}(1)^{*} \nu(F)\right|_{F_{l}},
$$

где $\psi_{l}(t)$ - ретракция нормальной $t$-трубки $N_{t}\left(F_{l}\right)$ на $F_{l}$. Следовательно, отображение $\alpha$ гомотопно отображению

$$
\beta(v)=\left\{\begin{array}{ll}
v, & \pi(v) \notin N\left(F_{l}\right), \\
\psi_{l}(|\pi \alpha(\pi(v))|)^{*}(v), & \pi(v) \in N\left(F_{l}\right),
\end{array} \quad l=1, \ldots, m .\right.
$$

Отметим, что построенное отображение есть результат малого шевеления вложения

$$
T \nu(F) \rightarrow T(\nu(F) \oplus \tau(F))
$$

при котором слои трубчатых окрестностей подмногообразий $F_{l}$ переходят в слои нормальных расслоений вложений $F_{l} \subset F$, соответствующих им при экспоненциальном отображении.

Построим отображение

$$
\tau(t)=\left(\beta(v) \oplus t \tilde{\alpha}_{*}(s(\pi(v)))\right)
$$

где $v \in T \nu(F), t \in[0 ; \infty)$, и устремим $t \mathrm{k} \infty$. Мы получим отображение $\tau(\infty)$, которое переводит в бесконечность точки расслоения $\nu(F)$, лежащие в слоях над внешностью трубчатых окрестностей подмногообразий $F_{l}$.

Слой трубчатой окрестности подмногообразия $F_{l}$ в $F$ под действием $\tau(\infty)$ отобразится на слой подрасслоения $\nu_{l}$ (см. вьше). При больших $t$ можно пренебречь первым слагаемым в сумме

$$
\beta(v) \oplus t \tilde{\alpha}_{*}(s(\pi(v)))
$$

Поэтому ограничение отображения $\tau(\infty)$ на слой трубчатой окрестности подмногообразия $F_{l}$ в $F$ совпадает с отображением $\phi_{l}^{s}$ (см. выше). Для завершения доказательства остается заметить, что образ отображения $\tau(\infty)$ совпадает с образом отображения $i$.

ЗАмЕчАниЕ 3.2. Требования, налагаемые на векторные поля в формулировке свойства локализации, можно ослабить. Достаточно потребовать, чтобы векторное поле было гомотопно полю Морса-Ботта в классе векторных полей с фиксированным множеством нулей.

ЗАмечАнИЕ 3.3. Нетрудно проверить, что если векторное поле $s$ на границе трубчатой окрестности подмногообразия нулей $F_{l}$ гомотопно полю внешних нормалей, то соответствующее этому подмногообразию отображение $j_{l}$ гомотопно тождественному отображению [8]. Эта ситуация выполняется всегда, когда векторное поле на многообразии задается действием окружности на этом многообразии [2]. 
ЗАмЕчАнИЕ 3.4. Непосредственно из доказательства теоремы 3.1 вытекает, что если касательное векторное поле на многообразии не имеет нулей, то трансфер этого многообразия гомотопен отображению в точку.

Свойство локализации и замечание 3.1 позволяют сразу получить следующее обобщение теоремы Пуанкаре-Хопфа (ср. [20]).

Теорема 3.2. Пусть $F$ - гладкое компактное замкнутое многообразие. Пусть на нем задано касательное векторное поле Морса-Ботта s. Предположим, что нули векторного поля $s$ образуют конечный набор связных компактных замкнутых подмногообразий $F_{1}, \ldots, F_{m}$ многообразия $F$. Тогда

$$
\chi(F)=\sum_{l=1}^{m} \epsilon\left(\operatorname{Ind}_{s}\left(F_{l}\right)\right) \cdot \chi\left(F_{l}\right),
$$

где $\chi(F)$ - эйлерова характеристика многообразия $F$.

ДоКАЗАТЕЛЬСтво. Мы будем использовать обозначения теоремы 3.1. Сначала рассмотрим произвольное касательное векторное поле на $F$ с изолированными невырожденными нулями. Применим свойство локализации к этому полю, получим

$$
I(F)=\{\pi \circ \tau\}=\sum_{k=1}^{N}\left\{\pi \circ j_{k}\right\}=\sum_{k=1}^{N} \epsilon\left(\operatorname{Ind}_{s}\left(z_{k}\right)\right),
$$

где $z_{k}$ - нули векторного поля, $k=1, \ldots, N$. В силу замечания 3.1 это означает, что $I(F)=\chi(F)$ - эйлерова характеристика многообразия $F$.

Применяя свойство локализации к полю $s$, получим

$$
\begin{aligned}
\chi(F) & =I(F)=\{\pi \circ \tau\}=\sum_{l=1}^{m}\left\{\pi \circ j_{l} \circ \tau_{l}\right\}=\sum_{l=1}^{m} \tau\left(p_{l}\right)^{*}\left\{\pi \circ j_{l}\right\} \\
& =\sum_{l=1}^{m} \epsilon\left(\operatorname{Ind}_{s}\left(F_{l}\right)\right) \cdot I\left(F_{l}\right)=\sum_{l=1}^{m} \epsilon\left(\operatorname{Ind}_{s}\left(F_{l}\right)\right) \cdot \chi\left(F_{l}\right) .
\end{aligned}
$$

\section{§4. Эквивариантные векторные поля и трансфер Беккера-Готтлиба}

В этом параграфе мы обобщим свойство локализации трансфера на случай произвольных расслоений с гладким слоем. Известные результаты из [5], [11] являются частными случаями нашего результата. В связи с этим мы показываем, как эти результаты могут быть получены в терминах введенного вьше индекса векторного поля.

Рассмотрим гладкое главное $G$-расслоение $(\mathscr{E}, G, B, p)$, база которого - компактное связное многообразие, а $G$ - компактная группа Ли. Пусть $F$ - компактное замкнутое связное многообразие с гладким действием группы $G$. Предположим, что на многообразии $F$ задано касательное векторное поле Морса-Ботта $s$, инвариантное относительно действия группы $G$. Нули векторного поля $s$ представляют собой объединение неприводимых инвариантных подмногообразий многообразия $F$. Обозначим их через $F_{1}, \ldots, F_{m}$. Заметим, что из неприводимости следует связность гладких многообразий

$$
E_{l}=\mathscr{E} \times{ }_{G} F_{l} .
$$


Всякое инвариантное относительно $G$ касательное векторное поле Морса-Ботта $s$ на $F$ позволяет нам построить векторное поле на $E=\mathscr{E} \times{ }_{G} F$, которое будет касательным вдоль слоев расслоения $E$. Будем обозначать его также через $s$. Имеем

$$
s: E \rightarrow \tau_{F}(E)=\mathscr{E} \times_{G} \tau(F) .
$$

Более того, векторное поле $s$ на $E$ также является полем Морса-Ботта. Нули поля $s$ на $E$ представляют собой подрасслоения $E_{l}=\mathscr{E} \times{ }_{G} F_{l}, l=1, \ldots, m$, расслоения $E$. Фиксируем вложение расслоения $E$ в $\mathbb{R}^{n}$. Точно так же, как и в предыдущем параграфе, векторное поле $s$ определяет отображения

$$
j_{l}: S^{n} \wedge E_{l}^{+} \rightarrow S^{n} \wedge E_{l}^{+} .
$$

Обозначим через $\tau\left(p_{l}\right)$ трансфер расслоения $E_{l}$. Пусть $\hat{g}$ - отображение, переводяшее $S^{n} \wedge B^{+}$в букет из $m$ пространств $S^{n} \wedge B^{+}$, индуцированное отображением $g: S^{n} \rightarrow \bigvee_{m} S^{n}$.

Теорема 4.1. Трансфер $\tau(p)$ расслоения Е гомотопически разлагается в композичию отображений

$$
\tau(p) \sim i \circ\left(j_{1} \circ \tau\left(p_{1}\right) \vee \cdots \vee j_{l} \circ \tau\left(p_{l}\right)\right) \circ \hat{g},
$$

где $і$ - вложсение пространства Тома тривиального п-мерного векторного расслоения над $E_{1} \cup \cdots \cup E_{m}$ в пространство Тома тривиального п-мерного векторного расслоения над $Е$.

ДокАЗАТЕЛЬСтво. Поскольку векторное поле $s$ инвариантно относительно действия группы $G$ на $F$, все гомотопии, описанные при доказательстве теоремы 3.1, эквивариантны относительно действия структурной групшы на слое. Поэтому имеет место эквивариантный аналог теоремы 3.1 , который и представляет собой формулировка теоремы 4.1 .

ЗАмечАниЕ 4.1. Требования, налагаемые на базу расслоения $E$, можно ослабить. Достаточно потребовать, чтобы база была клеточным комплексом. При этом снова достаточно провести доказательство для слоя расслоения, а затем воспользоваться его эквивариантностью относительно действия структурной группы расслоения. Заметим также, что для бесконечного клеточного комплекса гомотопическая эквивалентность понимается как эквивалентность стабильных классов отображений.

Пусть $h^{*}(\cdot)$ - мультипликативная теория когомологий с единицей. Обозначим через $\left(\operatorname{Ind}_{s}\left(E_{l}\right)\right)_{h}$ образ $\operatorname{Ind}_{s}\left(E_{l}\right)$ при естественном преобразовании теорий когомологий $\mu_{h}: \pi_{S}^{*} \rightarrow h^{*}$.

Теорема 4.2. В описанных выше условиях имеет место соотношение

$$
\tau(p)^{*} a=\sum_{i=1}^{m} \tau\left(p_{l}\right)^{*}\left(\left(\operatorname{Ind}_{s}\left(E_{l}\right)\right)_{h} \cdot i_{l}^{*} a\right),
$$

где $a \in h^{*}\left(E^{+}\right)$и $i_{l}: E_{l} \subset E-$ вложение расслоений, $l=1, \ldots, m$. 
ДокАЗАТЕЛЬСТво. Заметим, что отображения $j_{l}$ как элементы группы стабильно эквивалентных отображений из $E_{l}$ в себя однозначно определяют гомоморфизмы $j_{l}^{*}$ групп когомологий соответсвуюших пространств. Непосредственно из определения умножения в мультипликативных теориях когомологий следует, что гомоморфизм $j_{l}^{*}$ представляет собой умножение на $\left(\operatorname{Ind}_{s}\left(E_{l}\right)\right)_{h}$. Поэтому утверждение теоремы 4.2 следует из теоремы 4.1.

Теорема 4.2 позволяет получить результат работы [5] в следуюшем виде.

СлЕДСТВИЕ 4.1. Для гомоморфизмов теории сингулярных когомологий имеет место равенство

$$
\tau(p)^{*}=\sum_{l=1}^{m} \epsilon\left(\operatorname{Ind}_{s}\left(E_{l}\right)\right) \tau\left(p_{l}\right)^{*} i^{*}
$$

где $i: E_{l} \subset E, l=1, \ldots, m,-$ вложсение подрасслоений нулей поля $s$.

Для того чтобы получить из теоремы 4.2 результат работы [11], мы приведем сначала необходимые нам определения.

Рассмотрим главное $G$-расслоение $(\mathscr{E}, G, B, p)$, где $G$ - компактная группа Ли. Пусть $G$ действует на гладком замкнутом компактном многообразии $F$.

ОПРЕДЕЛЕНИЕ 4.1. Будем говорить, что орбиты точек $x_{1}, x_{2} \in F$ принадлежат одному типу, если их стабилизаторы $N\left(x_{1}\right), N\left(x_{2}\right)$ сопряжены. Тип орбиты точки $x_{1}$ меньше типа орбиты точки $x_{2}$, если $N\left(x_{2}\right)$ сопряжена подгруппе групшы $N\left(x_{1}\right)$.

Совокупность точек, принадлежаших орбитам одного и того же типа $\gamma$, образуют гладкое подмногообразие $F_{\gamma}$ в $F$, гранища которого принадлежит орбитам меньшего типа. Рассмотрим факторпространство $Y=F / G$. Замыкание множества $Y_{\gamma}=F_{\gamma} / G \subset Y$ является гладким многообразием с углами, и его граница лежит в $\bigcup_{\gamma^{\prime}<\gamma} Y_{\gamma^{\prime}}[18]$.

Tеорема 4.3 [18]. Существует симплициальное разбиение пространства $Y$, при котором внутренность каждого симплекса лежит в $\operatorname{Int}\left(Y_{\gamma}\right)$ для некоторого $\gamma$.

Покажем, как с помощью теоремы 4.1 можно доказать теорему 5.14 из [11].

ТЕОРема 4.4. В описанных выце условиях для гомоморфизма в произвольной теории когомологий, индуцированного трансфером расслоения $\mathscr{E} \times_{G} F$, имеет место формула

$$
\tau(p)^{*}=\sum_{\sigma \subset Y}(-1)^{\operatorname{dim} \sigma} \tau\left(p_{\sigma}\right)^{*}
$$

где $\tau\left(p_{\sigma}\right)$ - трансфер расслоения $\mathscr{E} \times_{G}\left(G / N\left(x_{\sigma}\right)\right), x_{\sigma}$ - произвольный прообраз барицентра симплекса $\sigma \subset Y$ в построенном в теореме 4.3 симплициальном разбиении. 
ДокАЗАТЕЛЬСТво. Построим на многообразии $F$ каноническое касательное векторное поле Морса-Ботта. Для этого сначала построим морсовское векторное поле на каждом $n$-мерном остове $\mathrm{sk}^{n} Y$ в симплициальном разбиении пространства $Y$. Построение проведем индукцией по размерности остова. Положим нулями поля $s$ все вершины симплексов симплициального разбиения. Нa $\mathrm{sk}^{1} Y$ определим векторное поле $s$ отталкиваюшимся из $\mathrm{sk}^{0} Y$ и притягивающимся в барицентры одномерных симплексов. Предположим, что мы построили векторное поле на $\operatorname{sk}^{n} Y$. Продолжим его до поля на $\mathrm{sk}^{n+1} Y$, полагая барищентры $(n+1)$-мерных симплексов притягиваюшими нулями поля $s$. Это завершает шаг индукции, а вместе с ним и построение поля. Таким образом, мы построили морсовское векторное поле на каждом остове $\operatorname{sk}^{n} Y$, нули которого суть все барицентры симплексов. Индекс нуля в барицентре симплекса размерности $n$ равен $(-1)^{n}$. Отображение $g: F \rightarrow F / G=Y$ для каждого симплекса $\sigma \subset Y$ индуцирует тривиальное расслоение $g: g^{-1}(\operatorname{Int}(\sigma)) \rightarrow \operatorname{Int}(\sigma)$. Фиксируем инвариантную относительно $G$ риманову метрику на многообразии $F$. Каноническое расщепление касательного расслоения

$$
\tau\left(g^{-1}(\operatorname{Int}(\sigma))\right)=g^{*} \tau(\operatorname{Int}(\sigma)) \oplus \operatorname{Ker} g_{*}
$$

позволяет поднять векторное поле $s$ на многообразие $g^{-1}(\operatorname{Int}(\sigma))$. Это поднятие будет согласованным для различных симплексов, поскольку

$$
\partial g^{-1}(\sigma)=g^{-1}(\partial \sigma)
$$

Обозначим поднятие поля $s$ на все $F$ через $\hat{s}$. Векторное поле $\hat{s}$ есть векторное поле Морса-Ботта на $F$, инвариантное относительно действия $G$ на $F$. Нулями векторного поля $\hat{s}$ будут подмногообразия $G / N\left(x_{\sigma}\right)$. Заметим, что

$$
\mathscr{E} \times_{G} g^{-1}(\sigma) \cong\left(\mathscr{E} \times_{G} G / N\left(x_{\sigma}\right)\right) \times \sigma
$$

Это означает, что нормальное расслоение вложения

$$
\left(\mathscr{E} \times_{G} G / N\left(x_{\sigma}\right)\right) \subset \mathscr{E} \times_{G} F
$$

есть тривиальное расслоение. Поэтому $\operatorname{Ind}_{\hat{s}}\left(G / N\left(x_{\sigma}\right)\right)=(-1)^{n}$, где $n$ - размерность симплекса $\sigma$. Отсюда вытекает утверждение теоремы.

ПримеР 4.1 (ср. [10]). Рассмотрим расслоение бутылки Клейна $K L$ над окружностью $S^{1}$ со слоем $S^{1}$ :

$$
p: K L \stackrel{S^{1}}{\longrightarrow} S^{1} .
$$

Индекс $I(p)$ построенного расслоения равен образующей групшы

$$
Z_{2} \cong \pi_{1}^{S} \subset \pi_{S}^{0}\left(S^{1+}\right)
$$


ДокАЗАТЕЛЬСТво. Пусть $\xi_{1}$ - нетривиальное одномерное вещественное векторное расслоение над $S^{1}$. Тогда расслоение $p: K L \rightarrow S^{1}$ изоморфно расслоению $p: R P\left(\xi_{1} \oplus 1\right) \rightarrow S^{1}$. Структурная группа этого расслоения действует на слое $S^{1}$ отражениями относительно фиксированной прямой. Неподвижные точки действия соответствуют двум подрасслоениям $R P\left(\xi_{1}\right)$ и $R P(1)$ в расслоении $R P\left(\xi_{1} \oplus 1\right)$. Нормальные расслоения вложений $R P\left(\xi_{1}\right), R P(1) \subset R P\left(\xi_{1} \oplus 1\right)$ изоморфны расслоению $\xi_{1} \otimes 1 \cong \xi_{1}$. Построим векторное поле на $S^{1}$, инвариантное относительно действия структурной групп расслоения $R P\left(\xi_{1} \oplus 1\right)$. Нулями этого поля будут неподвижные точки действия. Поле будет выталкивающим в одном нуле и притягиваюшим в другом. Кроме того, поле будет симметрично относительно прямой, проходящей через неподвижные точки действия. Это поле определяет послойное векторное поле Морса-Ботта $s$ на $R P\left(\xi_{1} \oplus 1\right)$. Нулями этого поля будут подрасслоения $R P\left(\xi_{1}\right)$ и $R P(1)$, пространства которых есть сечения расслоения $R P\left(\xi_{1} \oplus 1\right)$, т.е. $S^{1}$. Свойство локализации (теорема 4.1 ), примененное к векторному полю $s$, дает

$$
I(p)=\operatorname{Ind}_{s}\left(R P\left(\xi_{1}\right)\right)+\operatorname{Ind}_{s}(R P(1)) .
$$

Выталкивающий нуль поля $s$ имеет индекс, равньй 1 . Притягиваюший нуль имеет индекс, как в примере 3.2 , т.е. $-1+u$, где $u$ - образующая группы $\pi_{1}^{S}$. Следовательно, $I(p)=u$.

\section{§5. Векторные поля Морса-Ботта на грассманизациях разложимых векторных расслоений}

В этом параграфе мы построим послойное векторное поле Морса-Ботта награссманизации разложимого векторного расслоения и явно вычислим его индексы. Свойства этого векторного поля играют важную роль при доказательстве формул сложения для характеристических классов Понтрягина.

В дальднейшем будем обозначать через $R G_{k}^{n}$ многообразие Грассмана $k$-мерных подпространств $n$-мерного вешественного векторного пространства. Рассмотрим $n$-мерное вещественное векторное расслоение $\eta$ над базой $B$. Обозначим через $R G_{k}^{n}(\eta)$ ассоциированное с $\eta$ расслоение со слоем $R G_{k}^{n}$.

Пусть $\xi, \zeta$ - вешественные векторные расслоения над $B$ размерности $n_{1}, n_{2}$ соответственно $\left(n_{1}+n_{2}=n\right)$. Зададим действие группы положительных вещественных чисел $\mathbb{R}_{+}$на $\xi \times \zeta$ по формуле: $t \circ(v, u)=(v, t u)$. Это действие канонически переносится на грассманизацию $R G_{k}^{n}(\xi \times \zeta)$. Определим векторное поле $s$ :

$$
s(z)=\left.\frac{d}{d t}\right|_{t=1} t \circ z
$$

где $z \in R G_{k}^{n}(\xi \times \zeta)$, и покажем, что оно является полем Морса-Ботта. Для этого достаточно проверить, что на типичном слое $R G_{k}^{n}\left(\mathbb{R}^{n_{1}} \times \mathbb{R}^{n_{2}}\right)$ расслоения $R G_{k}^{n}(\xi \times \zeta)$ это поле есть поле Морса-Ботта.

Нули ограничения векторного поля $s$ на $R G_{k}^{n}\left(\mathbb{R}^{n_{1}} \times \mathbb{R}^{n_{2}}\right)$ суть неподвижные точки действия $\mathbb{R}_{+}$на этом слое, т.е. объединение многообразий $R G_{k_{1}}^{n_{1}}\left(\mathbb{R}^{n_{1}}\right) \times$ $R G_{k_{2}}^{n_{2}}\left(\mathbb{R}^{n_{2}}\right), k_{1}+k_{2}=k$ (см. [7]).

В окрестности точки $z_{0} \in R G_{k_{1}}^{n_{1}}\left(\mathbb{R}^{n_{1}}\right) \times R G_{k_{2}}^{n_{2}}\left(\mathbb{R}^{n_{2}}\right) \subset R G_{k}^{n}\left(\mathbb{R}^{n_{1}} \times \mathbb{R}^{n_{2}}\right)$ можно ввести систему координат:

$$
A(z)=\pi_{z}^{\perp} \circ \pi_{z}^{-1}
$$


где $A(z)$ - матрища размера $(n-k) \times k, \pi_{z}$ - ортогональная проекция плоскости $z$ на плоскость $z_{0}, \pi_{z}^{\perp}-$ ортогональная проекция плоскости $z$ на ортогональное дополнение к $z_{0}$ (окрестность точки $z_{0}$ состоит из всех точек $z$, для которых $\pi_{z}-$ изоморфизм). Поскольку плоскость $z_{0}$ есть прямая сумма плоскостей $x_{0} \in R G_{k_{1}}^{n_{1}}\left(\mathbb{R}^{n_{1}}\right)$ и $y_{0} \in R G_{k_{2}}^{n_{2}}\left(\mathbb{R}^{n_{2}}\right)$, то матрища $A(z)$ естественным образом разбивается на блоки:

$$
A(z)=\left(\begin{array}{ll}
D_{1} & D_{2} \\
D_{3} & D_{4}
\end{array}\right)
$$

где $D_{1}$ - матрица размера $\left(n_{1}-k_{1}\right) \times k_{1}, D_{2}$ - размера $\left(n_{1}-k_{1}\right) \times k_{2}, D_{3}$ - размера $\left(n_{2}-k_{2}\right) \times k_{1}, D_{4}$ - размера $\left(n_{2}-k_{2}\right) \times k_{2}$. Точки подмногообразия $R G_{k_{1}}^{n_{1}}\left(\mathbb{R}^{n_{1}}\right) \times$ $R G_{k_{2}}^{n_{2}}\left(\mathbb{R}^{n_{2}}\right)$ в этой окрестности выделяются уравнениями $D_{2}=D_{3}=0$, а точки слоя трубчатой окрестности этого же подмногообразия над точкой $z_{0}$ выделяются уравнениями $D_{1}=D_{4}=0$. В этой окрестности точки $z_{0}$ группа $\mathbb{R}_{+}$действует следуюшим образом:

$$
t \circ\left(\begin{array}{cc}
D_{1} & D_{2} \\
D_{3} & D_{4}
\end{array}\right)=\left(\begin{array}{cc}
D_{1} & D_{2} / t \\
t D_{3} & D_{4}
\end{array}\right) .
$$

Отсюда вытекает, что построенное векторное поле является полем МорсаБотта.

Опишем поведение векторного поля $s$ на всем пространстве $R G_{k}^{n}(\xi \times \zeta)$. Пусть $\mathscr{E}$ - главное $O(n)$-расслоение, ассоциированное с векторным расслоением $\xi \times \zeta$ над $B$. Нулями поля $s$ будут подрасслоения $R G_{k_{1}}^{n_{1}}(\xi) \times R G_{k_{2}}^{n_{2}}(\zeta), k_{1}+k_{2}=k$. Касательным расслоением вдоль слоев расслоения $R G_{k}^{n}(\xi \times \zeta)$ является векторное расслоение

$$
\mathscr{E} \times O(n) \tau\left(R G_{k}^{n}\right) .
$$

Пусть $\nu\left(R G_{k_{1}}^{n_{1}} \times R G_{k_{2}}^{n_{2}}\right)$ - нормальное расслоение вложения $R G_{k_{1}}^{n_{1}} \times R G_{k_{2}}^{n_{2}} \subset R G_{k}^{n}$. Оно изоморфно прямой сумме векторных расслоений $\xi_{n_{1}-k_{1}} \otimes \xi_{k_{2}}$ и $\xi_{k_{1}} \otimes \xi_{n_{2}-k_{2}}$, где через $\xi_{k}$ мы обозначаем $k$-мерное тавтологическое векторное расслоение над $R G_{k}^{n}, \quad \xi_{n-k}-(n-k)$-мерное векторное расслоение над $R G_{k_{2}}^{n_{2}}$, дополнительное к $\xi$ (отметим, что расслоение $\xi_{n-k}$ изоморфно $(n-k)$-мерному тавтологическому векторному расслоению над $\left.R G_{n-k}^{n}\right)$. Поэтому нормальное расслоение вложения

$$
R G_{k_{1}}^{n_{1}}(\xi) \times R G_{k_{2}}^{n_{2}}(\zeta) \subset R G_{k}^{n}(\xi \times \zeta)
$$

есть расслоение

$$
\nu=\mathscr{E} \times{ }_{O(n)} \nu\left(R G_{k_{1}}^{n_{1}} \times R G_{k_{2}}^{n_{2}}\right),
$$

и

$$
\nu \cong \pi_{1}^{*} \xi\left(k_{1}\right) \otimes \pi_{2}^{*} \zeta\left(n_{2}-k_{2}\right) \oplus \pi_{1}^{*} \xi\left(n_{1}-k_{1}\right) \otimes \pi_{2}^{*} \zeta\left(k_{2}\right),
$$

где через $\xi(k)$ мы обозначаем $k$-мерное тавтологическое векторное расслоение над $R G_{k}^{n}(\xi)$ (соответственно $\xi(n-k)$ есть $(n-k)$-мерное тавтологическое векторное расслоение над $\left.R G_{n-k}^{n}(\xi) \cong R G_{k}^{n}(\xi)\right), \pi_{1}, \pi_{2}$ - проекции на первый и второй множители в произведении $R G_{k_{1}}^{n_{1}}(\xi) \times R G_{k_{2}}^{n_{2}}(\zeta)$.

ЛЕмма 5.1. Векторное поле s сопоставляет подрасслоению $R G_{k_{1}}^{n_{1}}(\xi) \times$ $R G_{k_{2}}^{n_{2}}(\zeta)$ отображсение (см. $\left.\S 3\right)$

$$
\psi_{k_{1} k_{2}}^{s}: \nu \rightarrow \nu
$$


по формуле

$$
\phi_{k_{1} k_{2}}^{s}(u, v)=(u,-v),
$$

әде $u \in \pi_{1}^{*} \xi\left(k_{1}\right) \otimes \pi_{2}^{*} \zeta\left(n_{2}-k_{2}\right), \quad v \in \pi_{1}^{*} \xi\left(n_{1}-k_{1}\right) \otimes \pi_{2}^{*} \zeta\left(k_{2}\right)$.

ДокАЗАТЕльСтво. Достаточно проверить это утверждение на каждом слое расслоения $R G_{k_{1}}^{n_{1}}(\xi) \times R G_{k_{2}}^{n_{2}}(\zeta)$. На каждом слое этого расслоения в силу (5.1) ограничение $\phi_{k_{1} k_{2}}^{s}$ на слой есть симметрия относительно подрасслоения $\xi_{k_{1}} \otimes \xi_{n_{2}-k_{2}}$. Отсюда вытекает утверждение леммы.

СЛЕДСТВИЕ 5.1. В случае $k_{2}=0$ индекс векторного поля $s$ на подрасслоении нулей $R G_{k}^{n}(\xi) \times B$ равен 1 .

Доказательство очевидно.

СЛЕДСТВИЕ 5.2. Квадрат индекса $\operatorname{Ind}_{s}\left(R G_{k_{1}}^{n_{1}}(\xi) \times R G_{k_{2}}^{n_{2}}(\zeta)\right)$ нуля векторного поля $s$ равен 1 для любых $k_{1} u k_{2}$, где $k_{1}+k_{2}=k$.

ДокАЗАтельство. Заметим, что $\left(\psi_{k_{1} k_{2}}^{s}\right)^{2}=\mathrm{id}$ для всех $k_{1}$ и $k_{2}$, где $k_{1}+k_{2}=$ $k$. Поэтому утверждение следствия вытекает из определения умножения в кольце $\pi_{S}^{0}(\cdot)$ через композицию отображений.

\section{СЛЕДСТВИЕ 5.3. Имеет место равенство}

$$
\epsilon\left(\operatorname{Ind}_{s}\left(R G_{k_{1}}^{n_{1}}(\xi) \times R G_{k_{2}}^{n_{2}}(\zeta)\right)\right)=(-1)^{\left(n_{1}-k_{1}\right) k_{2}}
$$

Доказательство очевидно.

В заключение параграфа мы проведем вычисление индекса нуля векторного поля $s$ в случае, когда соответствующее ему отображение $\psi^{s}$ есть симметрия относительно некоторого подрасслоения. В качестве следствия мы получим явное выражение для индексов нулей поля $s$, построенного в этом параграфе.

В [6] рассмотрена теория кобордизмов $C O^{*}(\cdot)$, построенная по многообразиям, в стабильном касательном расслоении которых фиксирована структура комплексификации вещественного векторного расслоения. Любое расслоение вида $\mathbb{C} \otimes \xi$ ориентируемо в этой теории, т.е. обладает классом Тома. Для $n$-мерного векторного расслоения $\xi$ канонический класс Тома расслоения $\mathbb{C} \otimes \xi$ в этой теории определяется при помощи классифицирующего отображения

$$
u(\xi): T(\mathbb{C} \otimes \xi) \rightarrow T\left(\mathbb{C} \otimes \xi_{n}\right),
$$

где $\xi_{n}-n$-мерное тавтологическое векторное расслоение над $B O(n)$. Обозначим через $\bar{u}(\xi)$ другой класс Тома расслоения $\mathbb{C} \otimes \xi$ в этой теории, задаваемый при помощи отображения

$$
\bar{u}(\mathbb{C} \otimes \xi): T(\mathbb{C} \otimes \xi)=T(\xi \oplus \xi) \stackrel{(1,-1)}{\longrightarrow} T(\xi \oplus \xi)=T(\mathbb{C} \otimes \xi) \stackrel{u(\xi)}{\longrightarrow} T\left(\mathbb{C} \otimes \xi_{n}\right) .
$$

Пусть $\xi$ - вещественное векторное расслоение над конечным клеточным комплексом $B$ и $\xi^{\perp}-$ ортогональное дополнение к нему. Построим отображение

$$
\rho(\xi): T\left(\xi \oplus \xi^{\perp}\right) \stackrel{(-1,1)}{\longrightarrow} T\left(\xi \oplus \xi^{\perp}\right) .
$$

Обозначим через $\sigma_{m}$ изоморфизм надстройки $C O^{*}\left(B^{+}\right) \rightarrow C O^{*+m}\left(S^{m} \wedge B^{+}\right)$. 
ТЕОРЕМА 5.1. Для вещественного векторного расслоения $\xi$ в кольие $C O^{*}\left(B^{+}\right)$имеет место формула

$$
\sigma_{k}^{-1} \rho(\xi)^{*} \sigma_{k}(1)=u(\xi)^{-1} \bar{u}(\xi)
$$

где через $u^{-1}$ мы обозначаем обратное отображсение $\kappa$ изоморфизму Тома в теории $C O^{*}(\cdot)$, индуцированного классом Тома $u$, здесь $k=\operatorname{dim}\left(\xi \oplus \xi^{\perp}\right)$.

ДокАЗАтЕльство. Заметим, что отображение $\Sigma^{k}(\rho(\xi))$ гомотопно отображению

$$
T\left(\xi \oplus \xi \oplus \xi^{\perp} \oplus \xi^{\perp}\right) \stackrel{(1,-1,1,1)}{\longrightarrow} T\left(\xi \oplus \xi \oplus \xi^{\perp} \oplus \xi^{\perp}\right) .
$$

Поэтому утверждение теоремы немедленно следует из мультипликативности класca Тома.

Элемент (5.2) кольца $C O^{*}\left(B^{+}\right)$мы будем в дальнейшем обозначать через $\gamma(\xi)$.

Пусть $F_{l}$ - связная компонента многообразия нулей поля Морса-Ботта $s$ на многообразии $F$ и соответствующее отображение $\psi^{s}$ есть симметрия относительно некоторого подрасслоения. Пусть теперь $\xi-$ подрасслоение, на котором $\psi^{s}$ действует послойным умножением на -1 .

СлЕДСТВИЕ 5.4. Имеет место формула

$$
\left(\operatorname{Ind}_{s}\left(F_{l}\right)\right)_{C O}=\gamma(\xi)
$$

В частности, для векторного поля $s$ на грассманизации разложимого векторного расслоения, построенного выше в этом параграфе, выполняется равенство

$$
\left(\operatorname{Ind}\left(R G_{k_{1}}^{n_{1}}(\xi) \times R G_{k_{2}}^{n_{2}}(\zeta)\right)\right)_{C O}=\gamma\left(\pi_{1}^{*}\left(\xi\left(n_{1}-k_{1}\right)\right) \otimes \pi_{2}^{*}\left(\zeta\left(k_{2}\right)\right)\right)
$$

Рассмотрим формальную группу $f(u, v)=u+v+\sum_{i, j \geqslant 1} \alpha_{i j} u^{i} v^{j}$, где $\alpha_{i j} \in \Omega_{U}$, в комплексных кобордизмах [15]. Пусть $\bar{u} \in U^{2}(C P(\infty))$ - обратный элемент для $u \in U^{2}(C P(\infty))$ в этой группе, т.е. такой формальный ряд в $\Omega_{U}[[u]]$, что $f(u, \bar{u})=0$. Обозначим через $\phi(x)$ ряд такой, что $\bar{u}=u \phi(u)$, и $\phi_{n}\left(c_{1}, \ldots, c_{n}\right)-$ ряд в кольце $U^{*}(B U(n)) \cong \Omega_{U}\left[\left[c_{1}, \ldots, c_{1}\right]\right]$, задаваемьй тождеством

$$
\phi_{n}\left(\sigma_{1}\left(x_{1}, \ldots, x_{n}\right), \ldots, \sigma_{n}\left(x_{1}, \ldots, x_{n}\right)\right)=\prod_{i=1}^{n} \phi\left(x_{i}\right)
$$

где $\sigma_{i}$ - элементарные симметрические многочлены от $n$ переменных.

Используя каноническое преобразование $\mu_{U}^{C O}: C O^{*}(\cdot) \rightarrow U^{*}(\cdot)$, непосредственно из теоремы 5.1 получаем

СлЕДСТВИЕ 5.5. В кольие комплексных кобордизмов $U^{*}\left(B^{+}\right)$имеет место формула

$$
\left(\operatorname{Ind}_{s}\left(F_{l}\right)\right)_{U}=\gamma_{U}(\xi)=\phi_{n}\left(c_{1}(\mathbb{C} \xi), \ldots, c_{n}(\mathbb{C} \xi)\right),
$$

əде $\gamma_{U}(\xi)=\mu_{U}^{C O}(\gamma(\xi))$. 
СЛЕДСТВИЕ 5.6. Для одномерного вещественного векторного расслоения $\xi$ в кольче комплексных кобордизмов $U^{*}\left(B^{+}\right)$имеет место формула

$$
\gamma_{U}(\xi)=-1-\sum_{i, j \geqslant 1} \alpha_{i j}\left(c_{1}(\mathbb{C} \xi)\right)^{i+j-1}
$$

ДокАЗАТЕЛЬСТВО. Заметим, что $c_{1}(\mathbb{C} \xi)=c_{1}(\overline{\mathbb{C} \xi})$. Поэтому

$$
f\left(c_{1}(\mathbb{C} \xi), c_{1}(\mathbb{C} \xi)\right)=0 .
$$

Отсюда следует требуемое.

\section{§6. Теоремы сложения для характеристических классов Понтрягина}

В этом параграфе мы применим свойство локализации трансфера для изучения характеристических классов вешественных векторных расслоений, введенных в [6]. В случае обычных целочисленных когомологий эти классы с целыми номерами совпадают с классическими характеристическими классами Понтрягина, и потому они были названы в [6] также классами Понтрягина. Построение характеристических классов [6] опирается на следующие свойства трансфера (см. [3], [8]).

Пусть $\left(E_{i}, F_{i}, B_{i}, p\right), i=1,2,-$ расслоения, слои которых являются гладкими компактными замкнутыми многообразиями.

Свойство 6.1 (функториальность). Если

$$
\psi:\left(E_{1}, F_{1}, B_{1}, p\right) \rightarrow\left(E_{2}, F_{2}, B_{2}, p\right)
$$

- отображсение расслоений, то $\tau\left(p_{2}\right) \circ \psi \sim \psi \circ \tau\left(p_{1}\right)$.

Свойство 6.2 (мультипликативность). Если

$$
(E, F, B, p)=\left(E_{1} \times E_{2}, F_{1} \times F_{2}, B_{1} \times B_{2}, p_{1} \times p_{2}\right),
$$

mo $\tau\left(p_{1}\right) \wedge \tau\left(p_{2}\right) \sim \tau\left(p_{1} \times p_{2}\right)$.

Пусть $\xi$ - вешественное $n$-мерное векторное расслоение над конечным клеточным комплексом $B$ (без ограничения обшности можно считать, что $B$ - гладкое многообразие). Для данного $k, 1 \leqslant k \leqslant n$, рассмотрим грассманизацию векторного расслоения $\xi$, т.е. расслоение

$$
\left(R G_{k}^{n}(\xi), R G_{k}^{n}, B, p_{k}\right) .
$$

Пусть $\tau\left(p_{k}\right)$ - трансфер этого расслоения. Обозначим через $\xi(k)$ тавтологическое векторное расслоение над $R G_{k}^{n}(\xi)$ и через $f_{k}$ - классифицирующее его отображение $\xi(k)=f_{k}^{*} \xi_{k}$, где $\xi_{k}$ - тавтологическое расслоение над $B O(k)$. Пусть

$$
s_{0}: B O(k) \rightarrow T\left(\mathbb{C} \otimes \xi_{k}\right)
$$

- вложение на нулевое сечение. 
ОПРЕДЕЛЕНИЕ 6.1. Полуцелый класс Понтрягина $p_{k / 2}(\xi)$ в теории $C O^{*}$ равен

$$
p_{k / 2}(\xi)=\tau\left(p_{k}\right)^{*} f^{*} \chi\left(\mathbb{C} \otimes \xi_{k}\right)=\tau\left(p_{k}\right)^{*} f^{*}\left[s_{0}\right],
$$

где $\chi(\mathbb{C} \otimes \zeta)$ - эйлеров класс расслоения $\mathbb{C} \otimes \zeta$ в теории $C O^{*}$ (см. [6]). Положим $p_{k / 2}(\xi)=0$ при $k>n$.

Как следует из свойства трансфера 6.1, формула (6.1) задает характеристические классы вешественных векторных расслоений.

ТЕОРема 6.1. Пусть $\xi$ и $\zeta$ - вещественные векторные расслоения $(\operatorname{dim} \xi=$ $\left.n_{1}, \operatorname{dim} \zeta=n_{2}, n_{1}+n_{2}=n\right)$. Тогда для любого $k$ имеет место формула

$$
\begin{aligned}
p_{k / 2}(\xi \times \zeta)= & \sum_{k_{1}+k_{2}=k} \tau\left(p_{k_{1} k_{2}}\right)^{*}\left(\gamma\left(\pi_{1}^{*} \xi\left(n_{1}-k_{1}\right) \otimes \pi_{2}^{*} \zeta\left(k_{2}\right)\right)\right. \\
& \left.\times \chi\left(\mathbb{C} \otimes \xi\left(k_{1}\right)\right) \chi\left(\mathbb{C} \otimes \zeta\left(k_{2}\right)\right)\right),
\end{aligned}
$$

где $\tau\left(p_{k_{1} k_{2}}\right)$ - трансфер расслоения $R G_{k_{1}}^{n_{1}}(\xi) \times R G_{k_{2}}^{n_{2}}(\zeta), \pi_{1}$ и $\pi_{2}-$ проекиии на первый и второй сомножитель в произведении $R G_{k_{1}}^{n_{1}}(\xi) \times R G_{k_{2}}^{n_{2}}(\zeta)$.

ДокаЗАТЕЛЬСтво. Применим к векторному полю $s$ на $R G_{k}^{n}(\xi \times \zeta)$, построенному в $\S 5$, свойство локализации трансфера в форме теоремы 4.2 . В силу следствия 5.4 получаем

$$
\begin{aligned}
\tau\left(p_{k}\right)^{*} \chi(\mathbb{C} \otimes \eta(k))= & \sum_{k_{1}+k_{2}=k} \tau\left(p_{k_{1} k_{2}}\right)^{*}\left(\gamma\left(\pi_{1}^{*} \xi\left(n_{1}-k_{1}\right) \otimes \pi_{2}^{*} \zeta\left(k_{2}\right)\right)\right. \\
& \left.\times \chi\left(\mathbb{C} \otimes \xi\left(k_{1}\right)\right) \chi\left(\mathbb{C} \otimes \zeta\left(k_{2}\right)\right)\right),
\end{aligned}
$$

где через $\eta(k)$ мы обозначили $k$-мерное тавтологическое векторное расслоение над $R G_{k}^{n}(\xi \times \zeta)$. Остается заметить, что левая часть (6.2) по определению равна $p_{k / 2}(\xi \times \zeta)$.

Покажем, как из теоремы 6.1 может быть получена формула сложения для классов Понтрягина по модулю элементов 2-примарного порядка.

ТЕОРема 6.2. Пусть $\xi$ и нечной клеточной базой $B\left(\operatorname{dim} \xi=n_{1}, \operatorname{dim} \zeta=n_{2}, \quad n_{1}+n_{2}=n\right)$. Для любого $k$ имеет место формула

$$
p_{k / 2}(\xi \oplus \zeta)=\sum_{k_{1}+k_{2}=k}(-1)^{\left(n_{1}-k_{1}\right) k_{2}} p_{k_{1} / 2}(\xi) p_{k_{2} / 2}(\zeta) \quad(\bmod (2 \text { Tors })) .
$$

ДокАЗАТЕЛЬСТво. В силу функториальности классов Понтрягина достаточно доказать утверждение теоремы для расслоения $\pi_{1}^{*}(\xi) \oplus \pi_{2}^{*}(\zeta)$ над $B \times B$, где $\pi_{1}$ и $\pi_{2}-$ проекции на первый и второй сомножители в произведении $B \times B$. Заметим также, что

$$
\pi_{1}^{*}(\xi) \oplus \pi_{2}^{*}(\zeta) \cong \xi \times \zeta
$$

Положим

$$
\alpha=\left(\operatorname{Ind}_{s}\left(R G_{k_{1}}^{n_{1}}(\xi) \times R G_{k_{2}}^{n_{2}}(\zeta)\right)\right)_{C O} .
$$


В силу следствия $5.2 \alpha^{2}=1$. Имеем $\alpha= \pm 1+u$, где $u \in \widetilde{C O^{0}}\left(R G_{k_{1}}^{n_{1}}(\xi) \times\right.$ $\left.R G_{k_{2}}^{n_{2}}(\zeta)^{+}\right)$. Поскольку мы работаем в категории конечных клеточных комплексов, элемент $u$ нильпотентен. Соотношение $\alpha^{2}=1$ влечет равенство $\pm 2 u+u^{2}=0$. Отсюда и из нильпотентности $u$ заключаем, что элемент $u 2$-примарен, т.е. $u \in$ 2 Tors. Поэтому $\alpha= \pm 1(\bmod (2$ Tors $))$. В силу следствия 5.3

$$
\alpha \equiv(-1)^{\left(n_{1}-k_{1}\right) k_{2}} \quad(\bmod (2 \text { Tors })) .
$$

Обозначим через $\eta(k) \quad k$-мерное тавтологическое векторное расслоение над $R G_{k}^{n}(\xi \times \zeta)$. Из формулы $(6.2)$, учитывая (6.3), получаем

$$
\begin{aligned}
\tau\left(p_{k}\right)^{*} & \chi(\mathbb{C} \otimes \eta(k))= \\
& =\sum_{k_{1}+k_{2}=k}(-1)^{\left(n_{1}-k_{1}\right) k_{2}} \tau\left(p_{k_{1} k_{2}}\right)^{*}\left(\chi\left(\mathbb{C} \otimes \xi_{k_{1}}\right) \times \chi\left(\mathbb{C} \otimes \zeta_{k_{2}}\right)\right)(\bmod (2 \text { Tors })) .
\end{aligned}
$$

Из свойства мультипликативности трансфера 6.2 вытекает, что

$$
\begin{aligned}
& \tau\left(p_{k_{1}} \times p_{k_{2}}\right)^{*}\left(\chi\left(\mathbb{C} \otimes \xi\left(k_{1}\right)\right) \times \chi\left(\mathbb{C} \otimes \zeta\left(k_{2}\right)\right)\right) \\
& =\tau\left(p_{k_{1}}\right)^{*} \chi\left(\mathbb{C} \otimes \xi\left(k_{1}\right)\right) \tau\left(p_{k_{2}}\right)^{*} \chi\left(\mathbb{C} \otimes \zeta\left(k_{2}\right)\right) .
\end{aligned}
$$

Объединяя (6.4) и (6.5), получаем требуемую формулу:

$$
p_{k / 2}(\xi \times \zeta)=\sum_{k_{1}+k_{2}=k}(-1)^{\left(n_{1}-k_{1}\right) k_{2}} p_{k_{1} / 2}(\xi) \times p_{k_{2} / 2}(\zeta) \quad(\bmod (2 \text { Tors })) .
$$

ТеОРема 6.3. Получелье классы Понтрягина стабильны:

$$
p_{k / 2}(\xi \oplus 1)=p_{k / 2}(\xi)=p_{k / 2}(1 \oplus \xi) .
$$

ДокАЗАТЕЛЬСТво. Применим свойство локализации трансфера к расслоению $R G_{k}^{n+1}(\xi \oplus 1) \quad(\operatorname{dim} \xi=n)$. Используя следствием 5.1 , получим, что для любого элемента $x \in C O^{*}\left(R G_{k}^{n+1}(\xi \oplus 1)^{+}\right)$и некоторого элемента $\alpha \in$ $C O^{0}\left(R G_{k}^{n+1}(\xi \oplus 1)^{+}\right)$имеет место разложение

$$
\tau(p)^{*} x=\tau\left(p_{1}\right)^{*} i_{1}^{*} x+\tau\left(p_{2}\right)^{*} \alpha i_{2}^{*} x,
$$

где $\tau\left(p_{1}\right)$ - трансфер расслоения $\left(R G_{k}^{n}(\xi), R G_{k}^{n}, B, p_{1}\right)$ и $\tau\left(p_{2}\right)$ - трансфер расслоения $\left(R G_{k-1}^{n}(\xi), R G_{k-1}^{n}, B, p_{2}\right)$, остальные отображения определены в свойстве локализации.

Поскольку при вложении $R G_{k-1}^{n}(\xi) \subset R G_{k}^{n+1}(\xi \oplus 1)$ тавтологическое векторное расслоение $\xi(k)$ над $R G_{k}^{n+1}(\xi \oplus 1)$ переходит в сумму $\xi(k-1) \oplus 1$, где $\xi(k-1)$ тавтологическое векторное расслоение над $R G_{k-1}^{n}(\xi)$, то

$$
\begin{aligned}
\tau(p)^{*} \chi(\mathbb{C} \otimes \xi(k)) & =\tau\left(p_{1}\right)^{*} \chi(\mathbb{C} \otimes \xi(k))+\tau\left(p_{2}\right)^{*} \alpha \chi(\mathbb{C} \otimes(\xi(k-1) \oplus 1)) \\
& =\tau\left(p_{1}\right)^{*} \chi(\mathbb{C} \otimes \xi(k)) .
\end{aligned}
$$

Отсюда получаем первое равенство теоремы.

Для доказательства второго равенства необходимо векторное поле из $\S 5$ умножить на -1 и провести аналогичные рассуждения. 
СлеДСТвИЕ 6.1. При нечетных $k$ полуцелый класс Понтрягина $p_{k / 2}(\xi)$ 2-примарен.

ДокАЗАТЕЛЬСТВО. В силу теоремы 6.2

$$
p_{k / 2}(1 \oplus \xi)=(-1)^{k} p_{k / 2}(\xi) \quad(\bmod (2 \text { Tors })),
$$

а в силу теоремы 6.3

$$
p_{k / 2}(1 \oplus \xi)=p_{k / 2}(\xi) .
$$

При нечетных $k$ эти равенства совместны, если $p_{k / 2}(\xi) \in 2$ Tors.

СлЕДСТВИЕ 6.2. Для любых двух вещественных векторных расслоений $\xi$ и

$$
p_{2 k / 2}(\xi \oplus \zeta)=\sum_{k_{1}+k_{2}=k} p_{2 k_{1} / 2}(\xi) p_{2 k_{2} / 2}(\zeta) \quad(\bmod (2 \text { Tors })) .
$$

ТЕОРема 6.4. Для образа характеристических классов Понтрягина в комплексных кобордизмах существует формула, выражающая полущелье классы Понтрягина суммы двух вещественных векторных расслоений через характеристические классы слагаемых.

ДокАЗАТЕЛЬСТво. В силу разложения (6.2) достаточно показать, что $\left(\operatorname{Ind}_{s}\left(R G_{k_{1}}^{n_{1}}(\xi) \times R G_{k_{2}}^{n_{2}}(\zeta)\right)\right)_{U}$ может быть представлен в виде полинома от характеристических классов векторных расслоений $\xi\left(k_{1}\right)$ и $\zeta\left(k_{2}\right)$ с коэффишиентами в $p_{1}^{*}\left(U^{*}(B)\right) \otimes p_{2}^{*}\left(U^{*}(B)\right)$, где $p_{1}$ и $p_{2}-$ проекции в расслоениях $R G_{k_{1}}^{n_{1}}(\xi)$ и $R G_{k_{2}}^{n_{2}}(\zeta)$ соответственно. В силу леммы 5.1 и следствия 5.5 в комплексных кобордизмах имеет место равенство

$$
\left(\operatorname{Ind}_{s}\left(R G_{k_{1}}^{n_{1}}(\xi) \times R G_{k_{2}}^{n_{2}}(\zeta)\right)\right)_{U}=\phi_{\left(n_{1}-k_{1}\right) k_{2}}\left(c_{1}(\eta), \ldots, c_{\left(n_{1}-k_{1}\right) k_{2}}(\eta)\right),
$$

где $\eta \cong \mathbb{C} \otimes\left(\xi\left(n_{1}-k_{1}\right) \otimes \zeta\left(k_{2}\right)\right)$. Используя приншип расщепления для комплексных векторных расслоений и формулу для формальной группы

$$
f\left(c_{1}\left(\eta_{1}\right), c_{1}\left(\eta_{2}\right)\right)=c_{1}\left(\eta_{1} \otimes \eta_{2}\right),
$$

получаем выражение классов $c_{i}(\eta)$ через характеристические классы векторных расслоений $\mathbb{C} \otimes \xi\left(n_{1}-k_{1}\right)$ и $\mathbb{C} \otimes \zeta\left(k_{2}\right)$. Остается заметить, что $\xi\left(n_{1}-k_{1}\right) \oplus$ $\xi\left(k_{1}\right) \cong p_{1}^{*} \xi$.

ЗАмечАниЕ 6.1 . Полуцелые классы Понтрягина $p_{k / 2}(\xi)$ с нечетными номерами $k$ могут иметь любой 2-примарный порядок. Пусть, например, $\xi_{1}$ - одномерное тавтологическое векторное расслоение над $R P^{2 n+1}$. Обозначим через $\mu_{K}^{C O}: C O^{*}(\cdot) \rightarrow K^{*}(\cdot)$ естественное преобразование теорий когомологий. Порядок элемента $\mu_{K}^{C O}\left(p_{1 / 2}\left(\xi_{1}\right)\right) \in K^{*}\left(R P^{2 n+1}\right)$ равен $2^{n}$.

В заключение параграфа рассмотрим более детально теорему сложения для первого полуцелого класса Понтрягина (см. [9]).

Пусть $\xi$ и $\zeta$ - векторные расслоения над конечной клеточной базой $B$. Пусть $\tau(p)$ - трансфер расслоения $R P(\xi \oplus \zeta), \tau\left(p_{1}\right)$ - трансфер расслоения $R P(\xi), \tau\left(p_{2}\right)-$ трансфер расслоения $R P(\zeta)$. Обозначим через $i_{1}$ вложение $R P(\xi) \subset R P(\xi \oplus \zeta)$, через $i_{2}$ - вложение $R P(\zeta) \subset R P(\xi \oplus \zeta)$. 
Теорема 6.5. Для любого $x \in U^{*}(R P(\xi \oplus \zeta))$ имеет место формула

$$
\tau(p)^{*}(x)=\tau\left(p_{1}\right)^{*} i_{1}^{*}(x)+\tau\left(p_{2}\right)^{*}\left(\gamma_{U}\left(p_{2}^{*} \xi \otimes \zeta(1)\right) i_{2}^{*}(x)\right) .
$$

ДокАЗАТЕЛЬСТво. Утверждение теоремы немедленно следует из теоремы 4.2 и следствий 5.1 и 5.4 .

СлЕДСТВИЕ 6.3. Для любых двух одномерных вещественных векторных расслоений $\xi$ и Ц над конечной клеточной базой имеет место равенство

$$
p_{1 / 2}(\xi \oplus \zeta)=u-v-\sum_{i, j \geqslant 1} \alpha_{i j} f(u, v)^{i+j-1} v,
$$

где $u=p_{1 / 2}(\xi), v=p_{1 / 2}(\zeta)$.

ДоКАЗАТЕЛЬСТво. Для одномерного векторного расслоения $\xi$ имеем $p_{1 / 2}(\xi)=$ $c_{1}(\mathbb{C} \otimes \xi)$. Поэтому требуемое утверждение вытекает из явного вида $\gamma_{U}(\xi \otimes \zeta)$ (см. следствие 5.6).

Положим $[u]_{2}=u a(u)$, где $a(u)=2+\sum_{i, j \geqslant 1} \alpha_{i j} u^{i+j-1}$. Тогда результат следствия 6.3 можно переписать в виде

$$
p_{1 / 2}(\xi \oplus \zeta)=u+v-a(f(u, v)) v .
$$

Далее введем следуюшие симметричные по $u$ и $v$ ряды:

$$
\delta(u, v)=\frac{a(u)-a(v)}{u-v}, \quad d(u, v)=\frac{v a(u)-u a(v)}{u-v} .
$$

Запишем ряд $\alpha(u)=1+\sum_{i \geqslant 1} \alpha_{i 1} u^{i}$ в виде

$$
\alpha(u)=\alpha_{0}\left(u^{2}\right)+u \alpha_{1}\left(u^{2}\right) .
$$

ТЕорема 6.6. Для любых двух вещественных одномерных векторных расслоений $\xi$ и и имеет место равенство

$$
p_{1 / 2}(\xi \oplus \zeta)=u+v-u v\left[\alpha_{0}(u v) \delta(u, v)+\alpha_{1}(u v) d(u, v)\right],
$$

əде $u=p_{1 / 2}(\xi) u v=p_{1 / 2}(\zeta)$.

ДоКАЗАТЕЛЬСТвО. Положим

$$
\Phi(u, v)=1+\sum_{i, j \geqslant 1} \alpha_{i j} u^{i}\left(\frac{v^{j}-\bar{u}^{j}}{v-\bar{u}}\right) \in \Omega_{U}[[u, v]],
$$

где $\bar{u}: f(u, \bar{u})=0$. Тогда

$$
f(u, v)=f(u, v)-f(u, \bar{u})=(v-\bar{u}) \Phi(u, v) .
$$

Имеем

$$
f\left([u]_{2},[v]_{2}\right)=[f(u, v)]_{2}=f(u, v) a(f(u, v)) .
$$


Следовательно,

$$
\left([v]_{2}-[\bar{u}]_{2}\right) \Phi\left([u]_{2},[v]_{2}\right)=(v-\bar{u}) \Phi(u, v) a(f(u, v)) .
$$

Таким образом, в кольце $\Omega_{U}[[u, v]]$ имеет место тождество

$$
\Phi(u, v) a(f(u, v)) v=\left([v]_{2}+\bar{u} v \frac{a(v)-a(\bar{u})}{v-\bar{u}}\right) \Phi\left([u]_{2},[v]_{2}\right) .
$$

При доказательстве теоремы 6.6 достаточно считать, что $\xi$ и $\zeta$-одномерные тавтологические векторные расслоения над $R P(\infty)$. Поэтому далее все вычисления мы будем проводить в факторкольце

$$
A=\Omega_{U}[[u, v]] /\left([u]_{2},[v]_{2}\right) .
$$

Из тождества $[u]_{2}=(u-\bar{u}) \Phi(u, u)$ в $\Omega_{U}[[u]]$ немедленно следует, что в этом факторкольце $u=\bar{u}$ и $v=\bar{v}$. Используя соотношения $[u]_{2}=0$ и $u=\bar{u}$, мы получаем следуюшее тождество в $A$ :

$$
\Phi(u, v) a(f(u, v)) v=u v \delta(u, v),
$$

из которого непосредственно следует, что в $A$ имеет место соотношение

$$
a(f(u, v)) v=a(f(u, v)) u .
$$

Теперь, используя приведенный вьше явньй вид ряда $\Phi(u, v)$, получаем, что в кольце $A$ выполняется тождество

$$
a(f(u, v)) \Phi(u, v)=a(f(u, v)) \frac{\partial}{\partial v} f(u, v) .
$$

ЛЕмма 6.1. В кольце $\Omega_{U}[[u, v]]$ имеет место формула

$$
\frac{\partial}{\partial v} f(u, v)=\frac{C P(v)}{C P(f(u, v))}
$$

где $C P(v)=d g(v) / d v=1+\sum_{n \geqslant 1}[C P(n)] v^{n}$ u $g(v)$ - логарифм группь $f(u, v)$.

ДокАЗАТЕЛьСтво. В кольце $\Omega_{U} \otimes Q[[u, v]]$ для ряда $f(u, v)$ имеет место выражение

Так как

$$
f(u, v)=g^{-1}(g(u)+g(v)) .
$$

получаем, что

$$
\frac{d g^{-1}(x)}{d x} \cdot \frac{d g\left(g^{-1}(x)\right)}{d g^{-1}(x)}=1
$$

$$
\frac{\partial}{\partial v} f(u, v)=\frac{\partial g^{-1}(g(u)+g(v))}{\partial g(v)} \frac{\partial g(v)}{\partial v}=\frac{C P(v)}{C P(f(u, v))} .
$$

Возврашаясь к кольцу $A$, получаем

$$
a(f(u, v)) \frac{\partial}{\partial v} f(u, v)=a(f(u, v)) \frac{C P(v)}{C P(f(u, v))}=a(f(u, v)) C P(v) .
$$

Напомним, что

Следовательно,

$$
\frac{1}{C P(v)}=\left.\frac{\partial f(u, v)}{\partial u}\right|_{u=0}=1+\sum_{i \geqslant 1} \alpha_{i 1} v^{i}=\alpha(v) .
$$

$$
u v \delta(u, v) \alpha(v)=\alpha(v) \Phi(u, v) a(f(u, v)) v=a(f(u, v)) v .
$$


Лемма 6.2. В кольие А имеют место соотношения:

$$
\begin{aligned}
u v \delta(u, v) v & =u v d(u, v), \\
u v \delta(u, v) v^{2} & =u v \delta(u, v) u v .
\end{aligned}
$$

ДокАЗАТЕЛЬСтво. Первое равенство вытекает из соотношения

$$
\begin{aligned}
u v \frac{a(v)-a(u)}{v-u} v & =u v \frac{v a(v)-v a(u)+u a(v)-u a(v)}{v-u} \\
& =u v \frac{u a(v)-v a(u)+(v-u) a(v)}{v-u}=u v \frac{u a(v)-v a(u)}{v-u}=u v d(u, v) .
\end{aligned}
$$

Второе равенство следует из соотношения $a(f(u, v)) v=a(f(u, v)) u$ :

$$
u v \delta(u, v) v^{2}=\Phi(u, v) a(f(u, v)) v^{3}=\Phi(u, v) a(f(u, v)) v u v=u v \delta(u, v) .
$$

Применяя соотношения из леммы 6.2 , получаем

$$
\begin{aligned}
& u v \delta(u, v) \alpha(v)=u v \delta(u, v)\left(\alpha_{0}\left(v^{2}\right)+v \alpha_{1}\left(v^{2}\right)\right) \\
& =u v\left(\alpha_{0}(u v) \delta(u, v)+\alpha_{1}(u v) \delta(u, v) v\right)=u v\left(\alpha_{0}(u v) \delta(u, v)+\alpha_{1}(u v) d(u, v)\right),
\end{aligned}
$$

что завершает доказательство теоремы 6.6.

Эта теорема дает явный вид симметричного по $u$ и $v$ формального ряда

$$
b(u, v)=u+v+\sum_{i, j \geqslant 1} \beta_{i j} u^{i} v^{j}
$$

такого, что для любых двух одномерных вешественных векторных расслоений $\xi$ и $\zeta$ над $X$ имеет место формула

$$
p_{1 / 2}(\xi \oplus \zeta)=b(u, v) \in U^{2}(X)
$$

где $u=p_{1 / 2}(\xi)$ и $v=p_{1 / 2}(\zeta)$.

ТЕОРЕма 6.7 (формула сложения для первого полуцелого класса Понтрягина). Для любых двух вещественных векторных расслоений $\xi$ и

$$
p_{1 / 2}(\xi \oplus \zeta)=u+v+\sum_{k, l \geqslant 1} \beta_{k l} s_{k-1}(u) s_{l-1}(v)
$$

где $u=p_{1 / 2}(\xi), v=p_{1 / 2}(\zeta), s_{k}$ - операции Ландвебера-Новикова в комплексных кобордизмах [15]. 
ДокАЗАТЕЛЬСТВо. Из теоремы о локализации трансфера вытекает,что

$$
p_{1 / 2}(\xi \oplus \zeta)=p_{1 / 2}(\xi)+\tau(p)^{*}\left(\gamma_{U}\left(p^{*}(\xi) \otimes \zeta(1)\right) \cdot w\right)
$$

где $\tau(p)$ - трансфер расслоения $R P(\zeta), w=\chi(\mathbb{C} \otimes \zeta(1))$. Предположим сначала, что $\operatorname{dim} \xi=1$. Имеем

$$
\begin{aligned}
& p_{1 / 2}(\xi \oplus \zeta)=u+\tau(p)^{*}\left(\gamma_{U}\left(p^{*} \xi \otimes \zeta(1)\right) w\right) \\
& p_{1 / 2}(\zeta \oplus \xi)=v+\gamma_{U}(\zeta \otimes \xi) u
\end{aligned}
$$

где $u=p_{1 / 2}(\xi), v=p_{1 / 2}(\zeta)$.

Заметим, что в силу следствия 5.6

$$
\begin{aligned}
\tau(p)^{*}\left(\gamma_{U}\left(p^{*} \xi \otimes \zeta(1)\right) w\right) & =-\tau(p)^{*}\left(w+\sum_{i, j \geqslant 1} \alpha_{i j} f\left(p^{*} u, w\right)^{i+j-1} w\right) \\
& =\tau(p)^{*}\left(p_{1 / 2}\left(p^{*} \xi \oplus \zeta(1)\right)-p_{1 / 2}\left(p^{*} \xi\right)\right)
\end{aligned}
$$

Операции Ландвебера-Новикова суть стабильные когомологические операции, поэтому они коммутируют с отображением $\tau(p)^{*}$. В силу теоремы 6.6 получаем, что выражение (6.8) равно

$$
\tau(p)^{*}\left(w+\sum_{k, l \geqslant 1} \beta_{k l}\left(p^{*} u\right)^{k} w^{l}\right)=v+\sum_{k, l \geqslant 1} \beta_{k l} u^{k} s_{l-1}(v) .
$$

Приравнивая теперь (6.6) и (6.7), получаем

$$
\gamma_{U}\left(\zeta \otimes \xi^{*}\right) \cdot u=u+\sum_{k, l \geqslant 1} \beta_{k l} u^{l} s_{k-1}(v) .
$$

Рассмотрим случай произвольного $\xi$. Имеем

$$
\begin{aligned}
& p_{1 / 2}(\xi \oplus \zeta)=u+\tau(p)^{*}\left(\gamma_{U}\left(p^{*}(\xi) \otimes \zeta(1)^{*}\right) w\right) \\
& \quad=u+\tau(p)^{*}\left(w+\sum_{k, l \geqslant 1} \beta_{k l} w^{l} p^{*} s_{k-1}(u)\right)=u+v+\sum_{k, l \geqslant 1} \beta_{k l} s_{l-1}(v) s_{k-1}(u) .
\end{aligned}
$$

Это завершает доказательство.

ТеОРема 6.8. Характеристический класс $p_{1 / 2}$ задает отображение

$$
p_{1 / 2}: K O(X) \rightarrow U^{2}(X)
$$

на образе которого имеется ассочиативная операция сложения

$$
x \oplus y=x+y+\sum_{k, l \geqslant 1} \beta_{k l} s_{l-1}(y) s_{k-1}(x) .
$$

Обратим внимание, что эта операция не сводится ни к какой формальной группе. 


\section{§7. Характеристические классы Понтрягина комплексных векторных расслоений}

Теорема 7.1. Пусть $\eta-n$-мерное комплексное векторное расслоение. Трансфер $\tau(p)$ расслоения $R G_{2 k+1}^{2 n}(\eta)$ гомотопен отображению в точку. Трансферы $\tau\left(p_{1}\right)$ и $\tau\left(p_{2}\right)$ расслоений $R G_{2 k}^{2 n}(\eta)$ и $C G_{k}^{n}(\eta)$ связаны соотношением

$$
\tau\left(p_{1}\right) \sim i \circ \tau\left(p_{2}\right)
$$

где $i: C G_{k}^{n}(\eta) \rightarrow R G_{2 k}^{2 n}(\eta)$ - каноническое вложение.

ДокАЗАтЕльство. Рассмотрим расслоение $R G_{k}^{2 n}(\eta) \stackrel{R G_{k}^{2 n}}{\longrightarrow} B$. На пространстве этого расслоения действует группа $U(1)=S^{1}$. Это действие индуцировано комплексной структурой в векторном расслоении $\eta$. Неподвижными точками этого действия будут такие плоскости $\alpha$, что для любого вектора $v \in \alpha$ вектор $i v$ принадлежит $\alpha$. Поскольку векторы $v$ и $i v$ линейно независимы над полем действительных чисел, неподвижные точки возникнут только в том случае, когда $k$ четно. Применяя свойство локализации к векторному полю, соответствуюшему этому действию, получим, что трансфер $\tau(p)$ расслоения $R G_{2 k+1}^{2 n}(\eta)$ гомотопен отображению в точку (см. замечание 3.4 ). В случае четного $k$ неподвижными точками действия будут плоскости, которые являются овеществлением $(k / 2)$-мерных комплексных подпространств расслоения $\eta$. Применяя свойство локализации, получим, что $\tau\left(p_{1}\right) \sim i \circ \tau\left(p_{2}\right)$.

СлЕДСТВИЕ 7.1. Если расслоение $\eta$ стабильно изоморфно комплексному векторному расслоению, то $p_{(2 k+1) / 2}(\eta)=0$.

ДокАЗАТЕльСТво. Пусть расслоение $\eta \oplus[N]$ допускает структуру комплексного векторного расслоения; тогда

$$
p_{(2 k+1) / 2}(\eta)=p_{(2 k+1) / 2}(\eta \oplus[N])=0 .
$$

Для теории комплексных самосопряженных кобордизмов $S C^{*}(\cdot)$ имеют место естественные преобразования теорий когомологий

$$
\mu_{S C}^{C O}: C O^{*}(\cdot) \rightarrow S C^{*}(\cdot) \quad \text { и } \quad \mu_{S C}^{S p}: S p^{*}(\cdot) \rightarrow S C^{*}(\cdot),
$$

где $S p^{*}(\cdot)$ - теория симплектических кобордизмов (см. [6]).

СлЕДСТВИЕ 7.2. Если векторное расслоение $\eta$ стабильно әквивалентно комплексному векторному расслоению, то образ $p_{2 k / 2}(\eta)$ в $S C^{*}(B)$ при преобразовании $\mu_{S p}^{C O}$ лежит в $\operatorname{Im} \mu_{S C}^{S p}\left(S p^{*}(B)\right) \subset S C^{*}(B)$.

ДокАЗАТЕЛЬСТво. Поскольку классы Понтрягина стабильны, без ограничения общности можно считать, что само расслоение $\eta$ допускает комплексную структуру. Пусть $\tau\left(p_{1}\right)$ - трансфер расслоения $R G_{2 k}^{2 n}(\eta)$ и $\tau\left(p_{2}\right)$ - трансфер расслоения $C G_{k}^{n}(\eta)$. Обозначим через $i: C G_{k}^{n} \rightarrow R G_{2 k}^{2 n}$ каноническое вложение. Из теоремы 7.1 получаем

$$
\begin{aligned}
\mu_{S C}^{C O}\left(p_{2 k / 2}(\eta)\right) & =\mu_{S C}^{C O} \tau\left(p_{1}\right)^{*} \chi(\mathbb{C} \otimes \xi(2 k)) \\
& =\mu_{S C}^{C O} \tau\left(p_{2}\right)^{*} i^{*} \chi(\mathbb{C} \otimes \xi(2 k))=\mu_{S C}^{C O} \tau\left(p_{2}\right)^{*} \chi(\mathbb{C} \otimes \eta(k)) \\
& =\tau\left(p_{2}\right)^{*} \mu_{S C}^{C O} \chi(\mathbb{C} \otimes \eta(k))=\tau\left(p_{2}\right)^{*} \chi(\eta(k) \oplus \overline{\eta(k)}),
\end{aligned}
$$


где $\xi(2 k)$ - $2 k$-мерное тавтологическое векторное расслоение над $R G_{2 k}^{2 n}(\eta)$ и $\eta(k)-$ $k$-мерное тавтологическое векторное расслоение над $C G_{k}^{n}(\eta)$. Заметим, что для любого комплексного векторного расслоения $\eta$ расслоение $\eta \oplus \bar{\eta}$ наделено канонической симплектической структурой. Следовательно, правая часть (7.1) лежит $\operatorname{Im} \mu_{S C}^{S p}\left(S p^{*}(B)\right)$.

\section{Список литературы}

1. Atiyah M.F. Characters and cohomology of finite groups // Publ. Math. I.H.E.S. 1961. № 9. P. 23-64.

2. Becker J. C. Characteristic classes and $K$-theory // Lecture Notes in Mathematics. V. 428. Springer-Verlag, 1974. P. 132-143.

3. Becker J. C., Gottlieb D. H. The transfer map and fiber bundles // Topology. 1975. V. 14. № 1. P. 1-12.

4. Bressler P., Evens S. The Shubert calculas, braid relations, and generalized cohomology // Trans. Amer. Math. Soc. 1990. V. 317. P. 799-811.

5. Brumfiel G., Madsen I. Evaluation of the transfer and the universal surgery classes // Inventiones math. 1976. V. 32. P. 133-169.

6. Бухштабер В. М. Топологические приложения теории двузначных формальных групп // Изв. АН СССР. Сер. матем. 1978. Т. 42. № 1. С. 130-184.

7. Бухштабер B. М. Характеристические классы в кобордизамах и приложения теории однозначных и двузначных групп // Итоги науки и техники. Современные проблемы математики. Фундаментальные направления. Т. 10. М.: ВИНИТИ, 1978. С. 5-178.

8. Бухштабер В. М. Трансфер Беккера-Готтлиба. Прилож. 3 в книге: Снэйт В. Алгебраический кобордизм и $K$-теория. М.: Мир, 1983.

9. Бухштабер В.М., Фельдман К. Э. Формула сложения для первого полуцелого класса Понтрягина в комплексных кобордизмах // УМН. 1997. Т. 52. №6. С. 151-152.

10. Dold $A$. The fixed point index of fibre-preserving maps // Inventiones math. 1974. V. 25. P. 281-297.

11. Feshbach M. The transfer and compact Lie groups // Trans. of the Am. Math. Soc. 1979. V. 251. P. 139-169.

12. Fulton W., MacPherson R. Characteristic classes of direct image bundles for covering maps // Annals of Mathematics. 1987. V. 125. P. 1-92.

13. Милнор Джс., Сташеф Дж. Характеристические классы. М.: Мир, 1979.

14. Милнор Дже., Уоллес А. Дифференциальная топология. Начальный курс. М.: Мир, 1972.

15. Новиков С. П. Методы алгебраической топологии с точки зрения теории кобордизмов // Изв. АН СССР. Сер. матем. 1967. Т. 31. № 4. С. 885-951.

16. Постников М. М. Введение в теорию Морса. М.: Наука, 1971.

17. Снәйт B. Алгебраический кобордизм и $K$-теория. М.: Мир, 1983.

18. Verona A. Triangulation of stratified fibre bundles // Manu. Math. 1979/80. V. 30. P. $425-445$.

19. Уайтхед Джс. Новейшие достижения в теории гомотопий. М.: Мир, 1974.

20. Witten E. Suppersymmetry and Morse theory // J. Differential Geometry. 1982. V. 17. P. 661-692.

Московский государственный

Поступило в редакцию

университет им. М.В. Ломоносова

29.I.1999 